\title{
Estudo Empírico sobre Adoção de SOA: Um Mapeamento Sistemático da Literatura
}

\author{
José Jorge Lima Dias Jr. ${ }^{1,2}$, Joyce Aline Pereira de Oliveira ${ }^{2}$, Silvio Romero de \\ Lemos Meira ${ }^{2}$ \\ ${ }^{1}$ CCAE- Universidade Federal da Paraíba (UFPB) \\ Rio Tinto - PB - Brasil \\ ${ }^{2}$ Centro de Informática - Universidade Federal de Pernambuco \\ Recife - PE - Brasil \\ jorge@dce.ufpb.br, japo@cin.ufpe.br, silvio@cesar.org.br
}

Resumo. Arquitetura Orientada a Serviços (SOA) surgiu como uma alternativa para as organizações que objetivam aumentar a qualidade dos seus sistemas, melhorando a flexibilidade destes e o alinhamento entre negócio e TI. Entretanto, sua adoção, em um contexto corporativo, não é uma tarefa fácil. Neste sentido, este artigo apresenta um Mapeamento Sistemático da Literatura sobre adoção de SOA na indústria para responder questões de pesquisa referentes às etapas, atividades, lições aprendidas, dificuldades, influências e domínios sobre adoção de SOA. Para tanto, um conjunto de 27 trabalhos, entre eles, 21 estudos de caso, foram analisados.

\begin{abstract}
Service Oriented Architecture (SOA) has emerged as an alternative for organizations that aims at increasing the quality of their systems, improving flexibility of them and the business and IT alignment. However, its adoption is not an easy task in an enterprise context. Thus, this paper presents a Systematic Literature Mapping about SOA adoption in the industry in order to answer research questions regarding the steps, activities, lessons learned, difficulties, influences and domains about SOA adoption. For this purpose, a set of 27 studies, among them, 21 case studies were analyzed.
\end{abstract}

\section{Introdução}

Nas últimas décadas, a globalização tem levado as organizações a procurarem novas formas de criação de valor e repensarem sobre suas fronteiras organizacionais [Jacobides and Billinger 2006; Österle et al. 2006]. Muitas empresas estão focadas em aumentar a flexibilidade de negócio e simplificar sua infraestrutura de TI a fim de satisfazer seus objetivos de negócio [Nasr et al. 2010]. Além disso, equipes de desenvolvimento procuram melhorar a qualidade dos seus sistemas, através da melhoria da manutenabilidade e reusabilidade de suas aplicações, diminuindo também o tempo e o custo de desenvolvimento destas. Diante este cenário, SOA (Service-oriented Architecture) surgiu como uma alternativa para se atingir tais objetivos, uma vez que busca o alinhamento entre o negócio e a TI através da disponibilização e reutilização de funcionalidades, chamadas de serviços, que estão sob domínios de proprietários distintos [OASIS 2006].

São várias as definições de SOA na literatura, que variam em relação ao cenário que está sendo utilizado ou a um fornecedor específico de produtos SOA. Neste 
trabalho, consideramos que SOA é uma estratégia que possui um escopo para toda a organização [Wang and Liao 2009], ou seja, quando se adota SOA, diferentes áreas dentro de uma organização são afetadas [Software AG 2005]: estrutura organizacional, pessoas, processos e tecnologias de fluxo de trabalho. Bieberstein et al. (2005) afirma que é necessário uma reestruturação organizacional, incluindo comportamento cultural e individual, e que a adoção deve ser bem planejada. Para isso, alguns modelos de maturidade SOA foram propostos na indústria a fim de auxiliar no planejamento sistemático desta adoção [Dias et al. 2012].

A popularidade do conceito de SOA motivou a busca de evidências empíricas sobrecomo esta estratégia vem sendo efetivamente adotada nas organizações, a fim de identificar as boas práticas, dificuldades, lições aprendidas, processos, atividades, entre outros. Neste sentido, este trabalho apresenta um mapeamento sistemático da literatura [Petersen et al. 2008] sobre adoção de SOA para identificar estes aspectos e responder questões de pesquisa específicas. A pesquisa considerou trabalhos empíricos publicados em meios científicos no âmbito da adoção de SOA.

O restante deste artigo é organizado da seguinte forma: a seção 2 apresenta trabalhos relacionados; a seção 3 explica o método de pesquisa que foi utilizado; a seção 4 apresenta os resultados do mapeamento sistemático; finalmente, a seção 5 discute algumas considerações finais.

\section{Trabalhos Relacionados}

Lee et al. (2010) realizou um estudo exploratório na literatura identificando vários fatores de sucesso na adoção de SOA. Após a identificação destes fatores,foram realizadas entrevistas com especialistas da área para validar e identificar outros fatores não encontrados..

Outra revisão da literatura foi realizada por Luthria and Rabhi (2009). Nesta revisão os autores analisaram os seguintes aspectos: o valor percebido de SOA para a organização; a estratégia organizacional; cultura ou contexto organizacional; estrutura organizacional; desafios de implementação; e governança e gerenciamento. Baseado nos resultados, os autores propõe um conjunto de novas questões de pesquisa que podem ser exploradas na área de SOA.

Khadka et al. (2013) também executou uma revisão sistemática da literatura para analisar como o processo de migração de sistemas legados para SOA estava sendo abordado na literatura. Nesta pesquisa foram identificados as técnicas e os métodos para executar a migração.

A pesquisa realizada neste artigo se difere dos outros três trabalhos citados acima, em relação às questões de pesquisa. O trabalho de Lee et al. (2009) se restringiu a buscar trabalhos que relatavam apenas fatores de sucesso, inclusive este termo foi utilizado em sua string de busca. O trabalho de Luthria and Rabhi (2009) se restringiu a alguns aspectos específicos das características da adoção. Já a pesquisa de Khadka et al. (2013) analisou apenas as técnicas e métodos utilizados para migrar sistemas legados para SOA. A pesquisa descrita neste artigo analisa trabalhos empíricos sobre adoção de SOA na indústria em um contexto mais amplo, identificando outros aspectos e respondendo questões como atividades, benefícios, lições aprendidas, dificuldades, fatores que influenciam a adoção e domínios que a adoção foi aplicada, independentemente de terem tido ou não sucesso. 


\section{Método de Pesquisa}

Para executar esta pesquisa foi utilizado um Mapeamento Sistemático (MS) da literatura. Este fornece um procedimento sistemático e objetivo para identificar a natureza e extensão de estudos empíricos que estão disponíveis a fim de responder questões de pesquisa. Este tipo de estudo permite categorizar relatos e resultados que foram publicados, fornecendo, no final, um sumário visual do mapa de seus resultados. Este tipo de metodologia provê uma visão geral da área, identificando e classificando o tipo de pesquisa e os resultados [Petersen et al. 2008].

A comunidade de engenharia de software experimental vem trabalhando para definir processos padronizados para condução de mapeamentos sistemáticos. Este esforço pode ser observado em Petersen et al. (2008). Este trabalho fornece um processo bem definido que serve como um ponto de partida para o nosso trabalho, assim como os guidelines de revisão sistemática da literatura de Kitchenham (2007). Como pode ser visto na Figura 1, o processo utilizado por este trabalho possui três principais fases: 1 Definição da pesquisa, 2 - Coleta de estudos e 3 - Síntese dos Resultados.

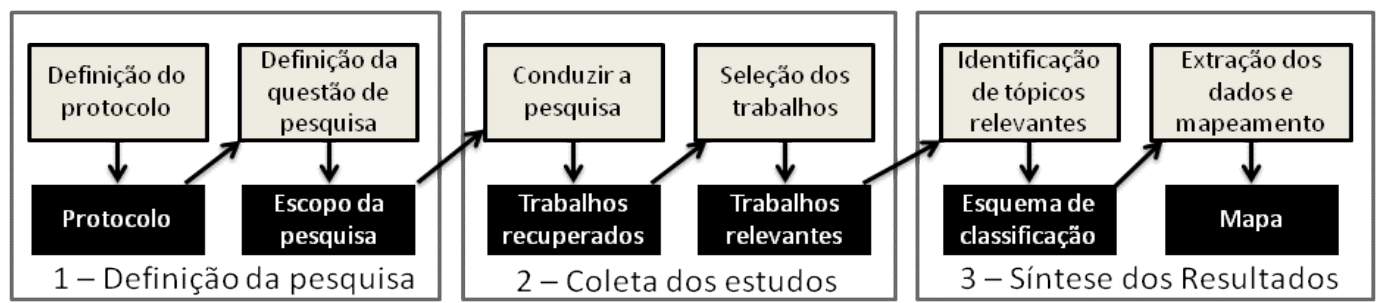

Figura 1. Processo de Mapeamento Sistemático (adaptado de Petersen et al. 2008)

$\mathrm{Na}$ Fase 1 foi elaborado um protocolo a fim de guiar toda a pesquisa. No protocolo definiram-se os objetivos da pesquisa, as questões que serão respondidas e todo o procedimento a ser executado no mapeamento. Na segunda fase foi executada a busca por estudos primários que estão de acordo com critérios bem definidos de inclusão e exclusão. Na terceira fase, os estudos primários selecionados, que satisfizeram os critérios, foram mapeados de acordo com um esquema de classificação.

\subsection{Definição da Pesquisa}

Uma revisão utilizando mapeamento sistemático requer um conjunto de fatos para diminuir o viés da pesquisa. Por isso, foi elaborado um protocolo contendo as questões de pesquisa, engenhos de busca a serem pesquisados, critérios de inclusão e exclusão e processo de extração dos dados. O protocolo aumenta a qualidade da pesquisa e ajuda a guiar os pesquisadores no processo de mapeamento. Revisões incrementais foram realizadas no protocolo durante a execução do mapeamento de acordo com os resultados obtidos nas buscas.

Como foi explicado anteriormente, o objetivo deste estudo é identificar, entender e caracterizar evidências relacionadas à adoção de SOA em estudos empíricos. Neste sentido, foram identificadas algumas questões de pesquisa específicas para ajudar na investigação deste processo de mapeamento:

\section{- QP1. Qual o processo de adoção utilizado?}


Objetivo: Identificar quais são as atividades, passos, fases, etc que foram definidas para a adoção de SOA. A ideia é investigar se existe um padrão de processo e as diferentes dimensões em que estão incluídas estas atividades.

- QP2. O que influencia a adoção de SOA?

Objetivo: Identificar quais são os principais fatores que podem impactar na adoção de SOA. É importante entender que fatores são estes a fim de institucionalizá-los em organizações que pretendem adotar SOA.

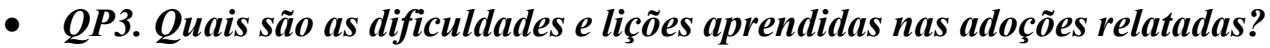

Objetivo: Descobrir as principais dificuldades enfrentadas na adoção de SOA a fim de permitir que possíveis soluções sejam propostas para resolver tais barreiras e facilitar a adoção.

- QP4. Quais foram os beneficios alcançados por quem adotou SOA?

Objetivo: Esta questão permitirá identificar os principais benefícios reportados na literatura, a fim de verificar se realmente estão de acordo com o que é prometido pela mídia sobre o assunto.

- QP5. Em que domínios SOA foi adotado?

Objetivo: A ideia desta questão é verificar em que domínios SOA tem sido adotado a fim de identificar se existe domínios mais propícios a adoção.

\subsection{Coleta dos Trabalhos}

Para a coleta dos estudos primários que irão fazer parte do mapeamento, é importante definir a estratégia de busca. Esta estratégia inclui a escolha de quais bibliotecas digitais serão utilizadas e que critérios de inclusão e exclusão serão considerados para análise.

Baseado nas questões de pesquisa, foram identificados os termos que serão utilizados na string de busca nas bibliotecas. Os dois principais termos que refletem o objetivo de pesquisa são: SOA e adoção. Para cada uma das palavras foram identificadas outras palavras chaves que remetem a elas, formando dois grupos:

- SOA: "SOA" / "service-oriented" / "service oriented" / "SOSE" / "SOC";

- Adoção: "adoption"/ "adopt" / "adopting" / "case study" / "case studies" / "roadmap".

Como o objetivo é identificar trabalhos que relatem a adoção de SOA na indústria, também foi utilizada a palavra-chave "case study".

Para montar a string de busca, que foi utilizada nas bibliotecas digitais de artigos científicos, utilizou-se o operador booleano OR entre as palavras do mesmo grupo e o operador AND para unir as palavras dos dois grupos.

A busca, utilizando a string acima, foi executada nas seguintes bibliotecas digitais: Scopus, IEEE Xplore e ACM Digital Library. Estes foram escolhidos por serem internacionalmente conhecidos pela qualidade dos estudos indexados. As buscas foram realizadas no período de 26 a 31 de julho de 2012. Portanto, este mapeamento está considerando apenas os trabalhos que já estavam indexados até este período.

A Figura 2 apresenta as atividades que foram executadas durante o processo de busca e seleção dos estudos e a quantidade de estudos que permaneceram no mapeamento depois de cada filtro. Na figura podemos perceber que 27 artigos permaneceram no mapeamento para serem analisados mais criticamente. 
Destes 27 trabalhos, 13 foram publicados em conferências internacionais (48\%), 6 em journals (22\%), e $8(30 \%)$ em conferências regionais (de um país, estado ou continente específico). É interessante destacar que os trabalhos foram publicados em 22 locais diferentes. Diante disto, é possível perceber a multidisciplinaridade de SOA, e que esta área pode ter diferentes focos.

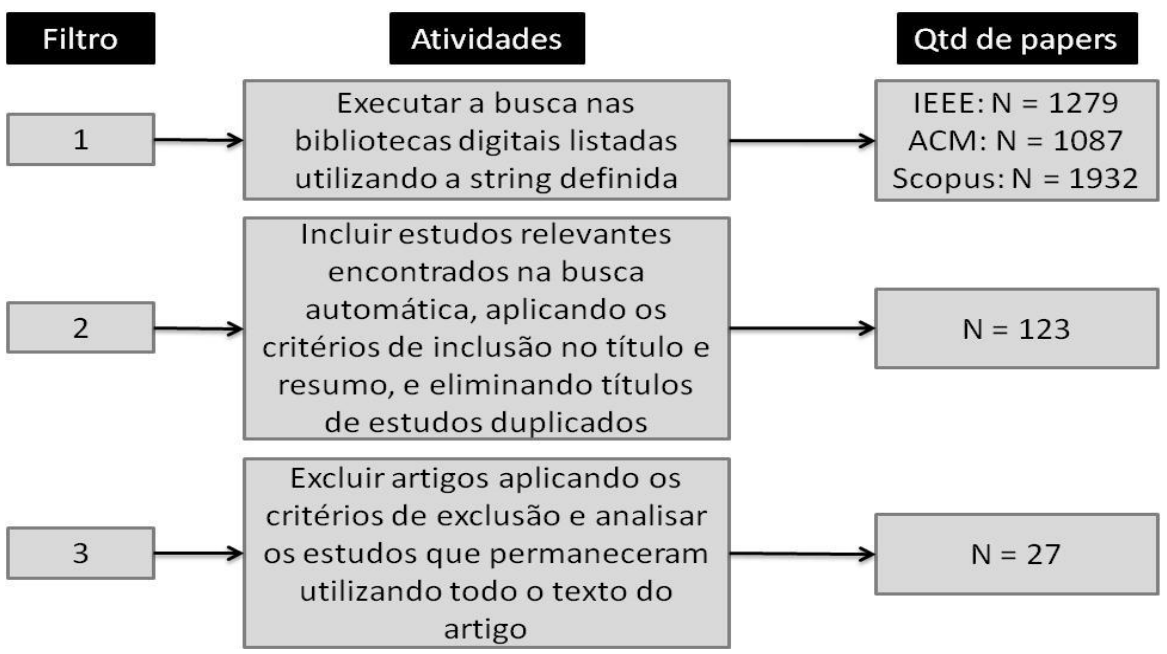

Figura 2. Estágios e resultados do processo de busca e seleção dos estudos

Os critérios de inclusão e exclusão foram definidos no protocolo e servem para analisar se um determinado estudo irá fazer parte ou não do mapeamento. Considerando os objetivos desta pesquisa, o seguinte critério de inclusão foi definido:

- Estudos primários que relatam, utilizando método empírico, a adoção de SOA corporativa, discutindo os aspectos da adoção.

Neste sentido, todos os artigos que satisfizeram este critério foram incluídos (filtro 2) para uma melhor análise. No filtro 3, os artigos incluídos foram analisados mais profundamente e então foram aplicados os critérios de exclusão:

- Trabalhos que relatam o desenvolvimento de um sistema utilizando SOA, sem considerar os aspectos de adoção SOA corporativa;

- Trabalhos que propõe resolver aspectos específicos sobre SOA sem discutir o processo de adoção;

- Estudos duplicados. Quando um estudo for publicado em mais de uma conferência, workshop ou periódico, a versão mais completa será considerada;

- Short papers com menos de três páginas;

- White papers, relatórios técnicos, livros e estudos secundários não serão considerados;

\section{Resultados}

Nesta seção serão apresentadas as descobertas referentes à cada questão de pesquisa, destacando as evidências identificadas no processo de extração dos dados. Por motivo de limite de espaço, nem todas as informações foram minunciosamente detalhadas, no entanto, o estudo completo poderá ser solicitado aos autores. $\mathrm{O}$ apêndice A mostra os 27 artigos primários que fizeram parte do mapeamento. 
A Figura 3 mostra que 21 dos estudos primários selecionados foram publicados entre 2009 e 2012 (77,8\% dos papers), o que pode evidenciar o quanto o tema em questão é atual. Esta porcentagem refere-se apenas a trabalhos empíricos pesquisados nas bases de busca citadas em 2.2. A baixa quantidade de artigos no ano de 2012 é justificada pelo período em que a busca foi realizada. Apesar da figura 3 indicar 2006 como ano inicial, a pesquisa não considerou como critério um período de tempo definido.

Quantidade de artigos por ano

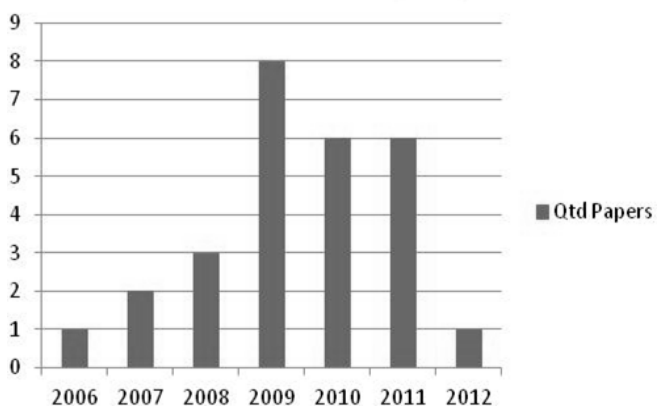

(a)

\section{Qtd de papers}

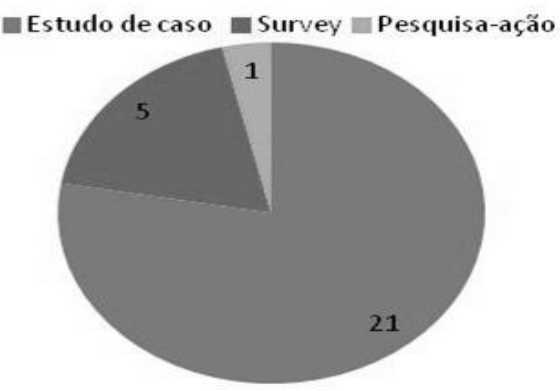

(b)

Figura 3. Quantidade de trabalhos por ano (3a) e Quantidade de trabalhos por método de pesquisa (3b)

A Figura 3 ainda mostra que $21(77,8 \%)$ utilizaram estudo de caso como método de pesquisa, $5(18,5 \%)$ realizaram survey, e apenas $1(3,7 \%)$ realizou uma pesquisa-ação (Figura 3b). Este é um aspecto importante do trabalho uma vez que os estudos de casos são, por definição, conduzidos em ambientes do mundo real e, portanto, possuem um alto grau de realismo (Runeson and Host 2009). Sendo assim, as descobertas através da análise destes estudos se tornam relevantes. Cada um destes trabalhos relatam um ou mais estudos de caso. Somando todos os estudos de caso, temos o total de 144 estudos de caso relatados nestes diferentes trabalhos. Não foram encontrados experimentos controlados o que pode evidenciar a ausência de utilização deste método no contexto de SOA.

As próximas subseções revisitarão as questões de pesquisa discutindo as descobertas identificadas.

\subsection{Fases e Atividades na adoção de SOA}

Esta subseção discute os processos de adoção identificados nos trabalhos analisados. Apenas 5 trabalhos (P1, P2, P4, P10, P23) apresentaram, explicitamente, de maneira sistemática, fases ou etapas na adoção de SOA.

Nos outros trabalhos não foi possível identificar estas etapas bem definidas, o que não significa que estas não existam, mas que não foram explicitadas no artigo analisado. $\mathrm{Na}$ análise destes trabalhos, percebe-se que alguns processos focam nos aspectos técnicos, como por exemplo, análise de tecnologias, identificação de serviços, integração de serviços, entre outros. Por outro lado, outros processos, também possuem etapas não técnicas, como por exemplo, definir governança SOA, definir centro de competência SOA, etc. O que os estudos mostram é que o processo de adoção varia caso a caso de acordo com a realidade da organização e o escopo de adoção. 
Analisando as diversas atividades que foram executadas nos trabalhos, percebeuse que elas se diferem muito em seus objetivos. Por este motivo, elas foram organizadas em quatro diferentes dimensões:

- Negócio: refere-se aos objetivos de negócio e escopo que precisam ser definidos na adoção de SOA;

- Arquitetura: refere-se à parte técnica de SOA;

- Processo: relacionado aos processos necessários ao se adotar SOA;

- Organização: se refere aos papéis, aspectos humanos e qualquer tipo de organização interna para se adaptar ao contexto de SOA.

$\mathrm{Na}$ Tabela 2 podemos verificar as atividades identificadas nos trabalhos categorizados de acordo com as dimensões acima. Destas atividades podemos destacar a definição do escopo onde é importante definir o que fará parte da adoção de SOA, que processos serão mapeados para serviços, e até onde SOA impactará no negócio. Na dimensão da Arquitetura, é importante analisar a situação atual de TI o que significa que é necessário entender os sistemas legados, identificando seus componentes e o que pode ser reutilizado como serviço. Na dimensão de Processo percebe-se a existência de dois ciclos de vida distintos, onde um é relacionado à concepção e implantação do serviço e outro relacionado a aplicação que reutiliza os serviços. Na dimensão de Organização destaca-se a criação de um centro de competência SOA. É este grupo que centraliza e toma decisões inerentes a adoção.

Tabela 2. Atividades identificadas nos estudos classificados por dimensão

\begin{tabular}{|c|c|c|c|}
\hline \multirow{6}{*}{ Negócio } & $\begin{array}{l}\text { Mapeamento dos processos de } \\
\text { negócio }\end{array}$ & \multirow{9}{*}{ Arquitetura } & $\begin{array}{l}\text { Definição do repositório de } \\
\text { serviços }\end{array}$ \\
\hline & Definição do escopo de adoção & & $\begin{array}{l}\text { Estabelecimento e teste do } \\
\text { barramento de servicos }\end{array}$ \\
\hline & Definição do apoio financeiro & & $\begin{array}{l}\text { Analisar situação de TI } \\
\text { existente }\end{array}$ \\
\hline & Identificação dos riscos & & Execução de projeto piloto \\
\hline & $\begin{array}{l}\text { Definição dos objetivos da } \\
\text { iniciativa }\end{array}$ & & $\begin{array}{l}\text { Definição de requisitos da } \\
\text { infraestrutura SOA }\end{array}$ \\
\hline & $\begin{array}{l}\text { Análise das necessidades de } \\
\text { negócio }\end{array}$ & & $\begin{array}{l}\text { Definição da estratégia de } \\
\text { transformar funcionalidades do } \\
\text { legado em serviços }\end{array}$ \\
\hline \multirow{6}{*}{ Processo } & $\begin{array}{l}\text { Definição do ciclo de vida do } \\
\text { serviço }\end{array}$ & & $\begin{array}{l}\text { Definição de arquitetura de } \\
\text { referência }\end{array}$ \\
\hline & $\begin{array}{l}\text { Definição do ciclo de vida da } \\
\text { aplicação (reutilizando os } \\
\text { serviços) }\end{array}$ & & Avaliação da arquitetura \\
\hline & $\begin{array}{l}\text { Definição de métricas para } \\
\text { avaliar a adoção de SOA }\end{array}$ & & Definição de padrões \\
\hline & $\begin{array}{l}\text { Definição de políticas e } \\
\text { governança SOA }\end{array}$ & \multirow{3}{*}{ Organização } & Definição de papéis \\
\hline & $\begin{array}{l}\text { Definição de um modelo de } \\
\text { maturidade SOA }\end{array}$ & & $\begin{array}{l}\text { Criação de um centro de } \\
\text { excelência SOA }\end{array}$ \\
\hline & Definição de um roadmap SOA & & Treinamento de pessoal \\
\hline
\end{tabular}


A frequência que cada atividade obteve na coleta dos dados não está sendo apresentada aqui, uma vez que o objetivo foi identificar todas as atividades possíveis que foram relatadas nos estudos analisados.

\subsection{Influências na adoção de SOA}

Esta questão de pesquisa tem como objetivo identificar quais são os fatores que podem influenciar positiva ou negativamente a adoção de SOA. Para analisar os artigos sob esta vertente, foram considerados fatores que os artigos relataram explicitamente como influências na adoção.

De acordo com as evidências mostradas na Tabela 3, dos fatores identificados, o que mais influencia na adoção de SOA é gerenciar os múltiplos times e unidades organizacionais (22,22\% dos trabalhos). Este aspecto inclui os problemas de integração destas unidades, as diferentes visões dos stakeholders, a quantidade e a confiança dos parceiros de negócio, além da pressão interna e externa gerada na organização.

Com 18,51\% aparecem os fatores Foco no processo e Complexidade tecnológica. O primeiro está relacionado com o enfoque dado pela organização à melhoria dos seus processos de negócio e com a capacidade destes serem implementados em forma de serviços. Esta é uma evidência que as áreas de SOA e BPM (Business Process Management) possuem um alinhamento importante. Já o segundo refere-se à seleção de tecnologias, ferramentas e abordagens necessárias para implementar SOA. Outro aspecto referente à complexidade tecnológica é o fato de se ter diversos sistemas heterogêneos.

Em seguida, apareceram, com 14,81\%, Tipo do projeto, Governança SOA e Estrutura organizacional como fatores que podem impactar na adoção. O primeiro refere-se às características do projeto que pode variar no seu escopo. Governança SOA refere-se às políticas e processos necessários para manter SOA em operação. A estrutura organizacional inclui vários aspectos, tais como: cultura, tipo, domínio, tamanho e organização da empresa.

Outros aspectos também foram citados com menor frequência, tais como expertise, treinamento e motivação para as equipes que trabalharem com SOA, e gerenciamento de riscos para identificar o que pode afetar o projeto negativamente.

Tabela 3. Mapeamento das evidências por fatores que influenciam na adoção

\begin{tabular}{|c|c|c|}
\hline Fatores que Influenciam na adoção & $\begin{array}{c}\text { Referências: Estudos } \\
\text { Primários (P) }\end{array}$ & $\begin{array}{c}\text { Quantidade de } \\
\text { trabalhos(\%) }\end{array}$ \\
\hline $\begin{array}{c}\text { Gerenciamento dos múltiplos times e } \\
\text { unidades }\end{array}$ & P6, P11, P16, P20, P26, P27 & $6(22,22 \%)$ \\
\hline Foco em processos (BPM) & P6, P17, P18, P21, P12 & $5(18,51 \%)$ \\
\hline Complexidade tecnológica & P6, P17, P18, P23, P27 & $5(18,51 \%)$ \\
\hline $\begin{array}{c}\text { Tipo do projeto (tamanho, custo, } \\
\text { complexidade) }\end{array}$ & P1, P20, P23, P27 & $4(14,81 \%)$ \\
\hline Governança SOA & P17, P22, P23, P26 & $4(14,81 \%)$ \\
\hline Estrutura organizacional & P9, P17, P23, P26 & $4(14,81 \%)$ \\
\hline Expertise, Treinamento e Motivação & P6, P17, P26 & $3(11,1 \%)$ \\
\hline Gerenciamento de riscos & P6, P27 & $2(7,4 \%)$ \\
\hline
\end{tabular}




\subsection{Dificuldades na adoção de SOA}

O objetivo desta questão é descobrir as principais dificuldades enfrentadas na adoção de SOA a fim de permitir que possíveis soluções sejam propostas para resolver tais barreiras e facilitar a adoção.

A Tabela 4 apresenta as dificuldades, desafios e preocupações que foram relatadas nos trabalhos analisados. A maior dificuldade relatada foi em relação à educação SOA $(25,92 \%)$, que inclui ausência de treinamento, falta de experiência nas tecnologias SOA, falta de habilidade nas ferramentas SOA, falta de profissionais SOA, entre outros. Governança SOA que inclui políticas, processos, etc, e reuso de serviços que inclui, que serviços construir para serem reutilizados e como construir serviços reutilizáveis, foi relatado por $22,22 \%$ dos trabalhos. Cinco trabalhos $(18,51 \%)$ relataram como dificuldade a definição de escopo, custo e esforço de projetos SOA, assim como também, os aspectos de segurança. A Cultura (18,51\%) é outro ponto considerado como desafio no processo de adoção, uma vez que as pessoas tem dificuldade de aceitar novas filosofias de trabalho.

Falta de maturidade dos sistemas legados também foi relatado como uma dificuldade por $14,81 \%$ dos trabalhos. Esta preocupação inclui complexidade destes sistemas legados, dificuldades em transformar funcionalidades do legado em serviços, ausência de suporte de legados a XML, entre outros. Gerenciamento de serviço surgiu como dificuldade em $14,81 \%$ dos trabalhos e inclui aspectos como gerir metadados, documentação, padronização, customização dos serviços e selecionar os melhores serviços que atendam aos requisitos. Com o mesmo quantitativo, granularidade dos serviços, também foi relatado como dificuldade ou desafio. Serviços podem ser compostos por outros serviços. Além disso, os serviços podem ser classificados de acordo com suas características operacionais com diferentes níveis de granularidade [Papazoglou, 2007]. Categorizar de maneira correta e encontrar o nível de granularidade correto potencializa a possibilidade de reuso destes serviços [Erl, 2005].

Outra dificuldade evidenciada é a ausência do envolvimento de pessoas de negócio $(11,11 \%)$, onde foi relatado, por exemplo, que falta o suporte destas pessoas ou que estas não participam do processo de desenvolvimento SOA.

A dificuldade em realizar testes $(11,11 \%)$ também foi evidenciada. Sai et al. (2006) relata algumas causas sobre a dificuldade de testar serviços: muitas vezes não se tem acesso ao código fonte uma vez que podem ser disponibilizados por entidades externas; serviços são compostos por outros serviços, rodando em várias máquinas com diferentes contextos; serviços podem ser descobertos em tempo de execução, o que dificulta predizer que serviços estão sendo executados no momento.

BPM também foi evidenciado como dificuldade $(11,11 \%)$. Novamente este resultado evidencia a forte relação das duas áreas (SOA e BPM). É importante que o planejamento da organização considere BPM caso tenha o objetivo de agilizar seus processos e obter o alinhamento entre TI e negócio.

Ausência de modelo de negócio e projeto de serviço também foram apareceram nos relatos, com 7,4\%. O primeiro refere-se à falta de processos de negócio modelados, o que dificulta a identificação dos serviços e o alinhamento de TI ao negócio. Projeto de serviço refere-se à especificação do design e arquitetura do serviço a ser desenvolvido, para que ele atenda os princípios de SOA [Erl, 2005]. 
Tabela 4. Mapeamento das evidências por dificuldades na adoção de SOA

\begin{tabular}{|c|c|c|}
\hline Dificuldades & Referências: Estudos Primários (P) & $\begin{array}{c}\text { Quantidade de } \\
\text { trabalhos (\%) }\end{array}$ \\
\hline Educação & P5, P8, P15, P16, P22, P23, P24, P25 & $8(25,92 \%)$ \\
\hline Governança SOA & P5, P8, P13, P15, P16, P23 & $6(22,22 \%)$ \\
\hline Reuso dos serviços & P8, P12, P13, P19, P21, P25 & $6(22,22 \%)$ \\
\hline $\begin{array}{c}\text { Definição de escopo, } \\
\text { custo, esforço e tempo } \\
\text { de projetos de SOA }\end{array}$ & P1, P5, P6, P12, P19 & $5(18,51 \%)$ \\
\hline Segurança & P6, P17, P22, P24, P25 & $5(18,51 \%)$ \\
\hline Cultura & P4, P5, P16, P22, P25 & $5(18,51 \%)$ \\
\hline $\begin{array}{c}\text { Falta de maturidade dos } \\
\text { sistemas legados }\end{array}$ & P4, P10, P17, P23 & $4(14,81 \%)$ \\
\hline $\begin{array}{c}\text { Granularidade dos } \\
\text { serviços }\end{array}$ & P8, P10, P17, P19 & $4(14,81 \%)$ \\
\hline $\begin{array}{c}\text { Gerenciamento do } \\
\text { serviço }\end{array}$ & P8, P10, P12, P22 & $4(14,81 \%)$ \\
\hline Imaturidade tecnológica & P5, P8, P23, P24 & $4(14,81 \%)$ \\
\hline Testes & P4, P8, P25 & $3(11,11 \%)$ \\
\hline $\begin{array}{c}\text { Falta de envolvimento } \\
\text { de pessoas de negócio }\end{array}$ & P4, P17, P25 & $3(11,11 \%)$ \\
\hline BPM & P8, P19, P21 & $3(11,11 \%)$ \\
\hline $\begin{array}{c}\text { Ausência de modelos de } \\
\text { negócio }\end{array}$ & P5, P8 & $2(7,4 \%)$ \\
\hline Projeto do serviço & P15, P23 & $2(7,4 \%)$ \\
\hline
\end{tabular}

\subsection{Lições aprendidas na adoção de SOA}

Referente a lições aprendidas (Tabela 5), foi evidenciada a importância de implantar Centros de Competência, ou seja, unidades funcionais responsáveis por gerir SOA, dentro da organização $(18,51 \%)$. Treinamentos, utilização de registro de serviços e governança foram outras lições aprendidas relatadas por $14,81 \%$ dos trabalhos. Registro de serviços é um repositório onde os serviços desenvolvidos podem ser armazenados, localizados e utilizados, potencializando o reuso.

Execução de projeto piloto apareceu com 11,1\%, mostra-se como uma estratégia que permite que os riscos sejam minimizados na adoção. Cultura de gestão por processos $(11,1 \%)$ evidencia que a área de BPM deve ser considerada ao se adotar SOA, quando o objetivo é alinhar a TI e o negócio ou agilizar os processos da organização. Utilizar um ESB (Enterprise Service Bus) também aparece com 11,1\%. ESB é um middleware que permite a integração de aplicações através de um barramento.

A criação de procedimentos e guias também apareceu como uma lição aprendida (7,4\%), o que confirma a importância de se ter uma governança SOA bem definida. 
Tabela 5. Mapeamento das evidências por lições aprendidas

\begin{tabular}{|c|c|c|}
\hline Lições aprendidas & $\begin{array}{c}\text { Referências: Estudos } \\
\text { Primários (P) }\end{array}$ & $\begin{array}{c}\text { Quantidade de } \\
\text { trabalhos (\%) }\end{array}$ \\
\hline $\begin{array}{c}\text { Criação de um Centro de Excelência } \\
\text { SOA }\end{array}$ & P4, P5, P15, P16, P26 & $5(18,51 \%)$ \\
\hline Treinamentos & P4, P8, P19, P21 & $4(14,81 \%)$ \\
\hline Utilização de registro de serviços & P8, P15, P17, P18 & $4(14,81 \%)$ \\
\hline Prática de Governança & P17 P19, P21, P26 & $4(14,81 \%)$ \\
\hline Execução de projeto piloto & P4, P8, P16 & $3(11,1 \%)$ \\
\hline Cultura de gestão por processos & P21, P19, P12 & $3(11,1 \%)$ \\
\hline Utilização de ESB & P4, P17, P18 & $3(11,1 \%)$ \\
\hline Criação de procedimentos e guias & P15, P16 & $2(7,4 \%)$ \\
\hline
\end{tabular}

\subsection{Benefícios alcançados na adoção de SOA}

Esta questão de pesquisa buscou responder quais são os reais benefícios atingidos pela adoção de SOA na indústria. A Tabela 6 apresenta estes benefícios.

Tabela 6. Mapeamento das evidências por benefícios alcançados

\begin{tabular}{|c|c|c|}
\hline Benefícios & Referências: Estudos Primários (P) & $\begin{array}{c}\text { Quantidade de } \\
\text { trabalhos (\%) }\end{array}$ \\
\hline Redução de custos & P4, P5, P10, P12, P13, P14, P18, P19, P23, & $10(37,04 \%)$ \\
\hline Pelhora na Integração & P11, P14, P18, P19, P24, P25, P27 & $7(25,92 \%)$ \\
\hline $\begin{array}{c}\text { Diminuição de tempo de } \\
\text { desenvolvimento }\end{array}$ & $\mathrm{P} 10, \mathrm{P} 18, \mathrm{P} 19, \mathrm{P} 22, \mathrm{P} 23, \mathrm{P} 24, \mathrm{P} 25$ & $7(25,92 \%)$ \\
\hline Agilidade organizacional & $\mathrm{P} 7, \mathrm{P} 8, \mathrm{P} 22, \mathrm{P} 24, \mathrm{P} 25, \mathrm{P} 27$ & $6(22,22 \%)$ \\
\hline $\begin{array}{c}\text { Melhora na } \\
\text { manutenabilidade }\end{array}$ & $\mathrm{P} 4, \mathrm{P} 10, \mathrm{P} 13, \mathrm{P} 23$ & $4(14,81 \%)$ \\
\hline Reusabilidade de serviços & $\mathrm{P} 22, \mathrm{P} 24, \mathrm{P} 25, \mathrm{P} 27$ & $4(14,81 \%)$ \\
\hline Aumento da Flexibilidade & $\mathrm{P} 14, \mathrm{P} 22, \mathrm{P} 25$ & $3(11,1 \%)$ \\
\hline $\begin{array}{c}\text { Redução do time-to- } \\
\text { market }\end{array}$ & $\mathrm{P} 7, \mathrm{P} 13, \mathrm{P} 23$ & $3(11,1 \%)$ \\
\hline
\end{tabular}

O benefício que mais foi relatado foi o de redução de custos $(37,04 \%)$, que pode incluir o custo de desenvolvimento ou de TI de maneira geral. Melhora da integração e diminuição no tempo de desenvolvimento também foram bastante relatados pelos trabalhos $(25,92 \%)$. A agilidade organizacional foi outro benefício citado em $22,22 \%$ dos trabalhos, alcançados pela melhoria na eficiência e na automatização dos processos. É importante dizer que este benefício é alcançado quando se considera também a área de BPM dentro de SOA.

Melhora na manutenabilidade e reusabilidade de serviços também foram relatados com 14,81\%. A comunicação entre consumidores e fornecedores de serviços é baseada em interfaces padronizadas, então a manutenabilidade dos sistemas é melhorada, uma vez que os detalhes de implementação são escondidas. Além disso, a capacidade de compor novos serviços a partir de outros já existentes facilita a manutenção, e aumenta a reusabilidade.

$\mathrm{O}$ aumento da flexibilidade e diminuição do time-to-market também foram relatados, ambos com 11,1\%. Aumentar a flexibilidade significa que os sistemas 
conseguem se adaptar mais rapidamente a novos requisitos. É uma característica que está associada com a melhora da manutenabilidade. A diminuição no time-to-market deve estar relacionada a outros benefícios alcançados como aumento da reusabilidade, melhoria na flexibilidade e manutenabilidade e redução do tempo de desenvolvimento.

Outros benefícios citados por trabalhos isolados foram: maior interação entre as unidades de negócio, visibilidade para os fluxos de negócio e satisfação do cliente.

É sabido que alguns benefícios estão relacionados e que um impacta consequentemente em outro. Por exemplo, a diminuição no tempo de desenvolvimento possivelmente melhora o time-to-market. $\mathrm{O}$ aumento da reusabilidade dos serviços, consequentemente pode diminuir o tempo e o custo de desenvolvimento. Entretanto, para consolidar estes resultados, e evitar viés na interpretação, foram utilizados os termos que os trabalhos utilizaram em seus respectivos trabalhos.

\subsection{Domínios de aplicação de SOA}

Considerando que em um trabalho pode conter o relato de um ou mais estudos de caso, cada trabalho também pode ter relatado um ou mais domínios. O domínio Financeiro foi o mais evidenciado, com 31,71\% (total de 13 trabalhos). O domínio de Governo apareceu em 6 trabalhos (14,63\%). Realmente é perceptível que em vários setores do governo brasileiro, por exemplo, possui um grande potencial para a adoção de SOA, uma vez que possui uma grande quantidade de sistemas heterogêneos que necessitam ser integrados. As áreas de Telecomunicações, Seguro e Saúde também apareceram entre os domínios que mais adotaram SOA. A Figura 4 resume os resultados nos diferentes domínios. Outros domínios que apareceram em menor número foram: comércio, logística, indústria e transporte.

Existem características em comum nos domínios identificados no mapeamento. Uma delas é o fato destes domínios possuírem processos de negócio bem definidos e delimitados. Considerando que serviços representam bem atividades destes processos [Erl, 2005], é factível que se busque a utilização de BPM (Business Process Management) aliada a SOA para melhorar estes processos.

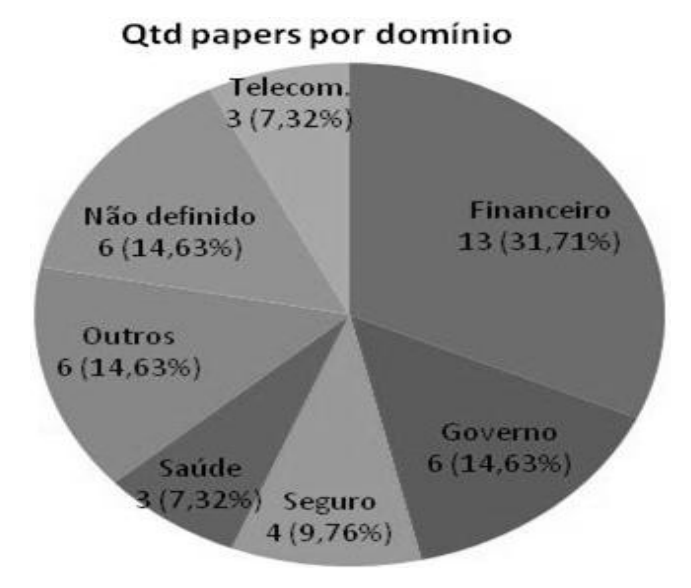

Figura 4. Quantidade de trabalhos por domínios

\subsection{Limitações do Trabalho}

Em qualquer processo de revisão ou mapeamento da literatura, não há garantia de que todos os estudos primários relevantes serão selecionados. É possível que alguns estudos 
primários relevantes não tenham sido identificados no processo de busca, seja devido à escolha dos termos utilizados na string de busca ou devido à limitação do próprio engenho de indexação e busca das bibliotecas digitais utilizadas.

Outra limitação do trabalho é a análise realizada nos artigos, pois depende muitas vezes da interpretação e experiência dos pesquisadores. Entretanto, isto pode ser minimizado através de um protocolo de pesquisa bem definido.

\section{Considerações Finais}

Este trabalho apresentou um mapeamento sistemático para identificar processos, atividades, lições aprendidas, dificuldades, benefícios e domínios referentes à adoção de SOA na indústria. A identificação destes elementos poderá auxiliar os profissionais e os pesquisadores da área a compreender o que deve ser considerado na adoção efetiva de SOA em um ambiente real e quais são as barreiras normalmente encontradas neste processo. O diferencial desta pesquisa é a quantidade de trabalhos que relatam estudos de caso na área, dando maior relevância ao resultado. Analisando as etapas e atividades identificadas nos trabalhos, pudemos organizá-las em quatro dimensões diferentes: Processo, Arquitetura, Negócio e Organização.

Sobre os fatores que impactam positiva ou negativamente a adoção de SOA destacou-se o gerenciamento dos múltiplos times e unidades organizacionais, o foco nos processos e a complexidade tecnológica envolvida. Em relação às dificuldades relatadas, a educação, a governança SOA e o reuso dos serviços se destacaram. Sobre as lições aprendidas foi evidenciada a importância da criação de um centro de competência SOA. Sobre os benefícios alcançados, a redução de custos em geral foi a mais apontada.

Percebeu-se que a adoção de SOA é ainda um tema que necessita de mais pesquisas e que as variáveis que estão associadas ao sucesso desta variam muito de acordo com o cenário e o escopo desta adoção. Portanto, outros tipos de pesquisa são necessários, diminuindo os riscos e melhorando a efetividade desta adoção.

\section{Referências}

Runeson, P; Host, M.(2009) "Guidelines for conducting and reporting case study research in software engineering, Empirical Software Engineering", v. 14, n. 2, 2009, pp. 131-161.

Kitchenham, B. A. (2007) "Guidelines for performing Systematic Literature Reviews in Software Engineering”, Version 2.3, EBSE Technical Report, EBSE-2007-01, 2007.

Petersen, K; Feldt, R; Mujtaba, S e Mattsson,M.(2008) "Systematic Mapping Studies in Software Engineering", 12th International Conference on Evaluation and Assessment in Software Engineering (EASE), pp. 26-27, University of Bari, Italy, June .

Nasr, K., Gross, H., Deursen, A. (2010) "Adopting and Evaluating Service Oriented Architecture in Industry". 14th European Conference on Software Maintenance and Reegineering.

Dias, J., Oliveira, J., Meira, S. (2012) "Pontos Chaves para Adoção de Uma Arquitetura Orientada a Serviços: Uma Análise Comparativa de Modelos de Maturidade SOA da Indústria”. Simpósio Brasileiro de Sistemas de Informação, São Paulo. 
Lee, J. H., Shim, H. J., Kim, K. K. (2010) "Critical Success Factors in SOA Implementation: An Exploratory Study". Journal Information Systems Management, Volume 27, Issue 2, pp. 123-145.

Österle, H., Fleisch, E., \& Alt, R. (2001). Business networking —shaping collaboration between enterprises (Second Revised and Extended Edition ed.) Springer.

Jacobides, M. G., \& Billinger, S. (2006). Designing the boundaries of the firm: from “ Make, Buy, or Ally" to the dynamic benefits of vertical architecture. Organization Science, 17(2), 249 - 261.

Software AG. , (2005) "SOA Governance Rule Your SOA", BP Trends.

OASIS (2006). "Reference Model For Service Oriented Architecture". Disponível em < http://www.oasis-open.org/committees/download.php/16587/wd-soa-rm-cd1ED.pdf $>$

Bieberstein, N; Bose, S; Walker,L e Lynch, A. (2005) "Impact of service-oriented architecture on enterprise systems, organizational structures, and individuals", IBM Systems Journal, VOL.44, NO.4, PP.691-708.

Luthria, H., Rabhi, F. (2009) "Service Oriented Computing in Practice - An Agenda for Research into the Factors Influencing the Organizational Adoption of Service Oriented Architectures". Journal of Theoretical and Applied Electronic Commerce Research, vol 4, Issue 1, pp. 39-56.

Wang, Y., Liao, J. (2009) "Why Or Why Not Service Oriented Architecture". International Conference on Services Science, Management and Engineering, pp. 6568.

Khadka, R., Saeidi, A., Idu, A., Hage, J., Jansen, J. (2013). "Legacy to SOA Evolution: A Systematic Literature Review". In A. D. Ionita, G. Lewis \& M. Litoiu (Eds.), Migrating Legacy Applications: Challenges in Service Oriented Architecture and Cloud Computing Environments: IGI Global.

Papazoglou, M. P. (2007). "What's in a Service?", 1st European Conference on Software Architecture. Proceedings in Springer. Madrid, Spain.

Erl, T. (2005) "Service-Oriented Architecture: Concepts, Technology, and Design", Prentice Hall PTR.

Sai, W. T., Gao, J., Wei, X., Chen, Y. (2006). "Testability of Software in ServiceOriented Architecture", 30th Annual International References 143 Computer Software and Applications Conference (COMPSAC'06). Chicago, USA, pp. 163170.

Apêndice A

\begin{tabular}{|c|l|l|c|}
\hline ID & Autores & Trabalho & Ano \\
\hline P1 & O'Brien, L. & $\begin{array}{l}\text { A Framework for Scope, Cost and Effort } \\
\text { Estimation for Service Oriented Architecture } \\
\text { (SOA) Projects. }\end{array}$ & 2009 \\
\hline P2 & $\begin{array}{l}\text { Reijnders, G.; Saeidi, A.; } \\
\text { Jansen, S.; Hage, J. }\end{array}$ & $\begin{array}{l}\text { A method engineering based legacy to SOA } \\
\text { migration method. }\end{array}$ & 2011 \\
\hline P3 & Razavian, M.; Lago, P. & A survey of SOA migration in industry & 2011 \\
\hline P4 & Nasr, K. A. & $\begin{array}{l}\text { Adopting and Evaluating Service Oriented } \\
\text { Architecture in Industry }\end{array}$ & 2010 \\
\hline P5 & Kokko, T., Antikainen, J., & Adopting SOA - Experiences from Nine Finnish & 2009 \\
\hline
\end{tabular}




\begin{tabular}{|c|c|c|c|}
\hline & Systa, T. & Organizations & \\
\hline P6 & Green, P., Baumgartner, I. & $\begin{array}{l}\text { Adoption of service oriented computing from the } \\
\text { IT professionals' perspective: An e-government } \\
\text { case study }\end{array}$ & 2008 \\
\hline P7 & $\begin{array}{l}\text { Chen, H., Perry, O., } \\
\text { Kazman, R. }\end{array}$ & $\begin{array}{l}\text { An integrated framework for service engineering: } \\
\text { a case study in the financial services industry }\end{array}$ & 2009 \\
\hline P8 & Brahe, S. & $\begin{array}{l}\text { BPM on top of SOA: Experiences from the } \\
\text { financial industry }\end{array}$ & 2007 \\
\hline P9 & Luthria, H., Rabhi, F. A. & $\begin{array}{l}\text { Building the Business Case for SOA: A Study of } \\
\text { the Business Drivers for Technology } \\
\text { Infrastructure Supporting Financial Service } \\
\text { Institutions }\end{array}$ & 2007 \\
\hline P10 & Tao, A. T., Yang, J. & $\begin{array}{l}\text { Develop service oriented finance business } \\
\text { processes: A case study in capital market }\end{array}$ & 2006 \\
\hline P11 & $\begin{array}{l}\text { Kumar, S., } \\
\text { Dakshinamoorthy, V., } \\
\text { Krishnan, M. S. }\end{array}$ & $\begin{array}{l}\text { Does SOA Improve the Supply Chain? An } \\
\text { Empirical Analysis of the Impact of SOA } \\
\text { Adoption on Electronic Supply Chain } \\
\text { Performance }\end{array}$ & 2007 \\
\hline $\mathrm{P} 12$ & $\begin{array}{l}\text { Auer, L., Belov, E., } \\
\text { Kryvinska, N., Strauss, C. }\end{array}$ & $\begin{array}{l}\text { Exploratory case study research on SOA } \\
\text { investment decision processes in Austria }\end{array}$ & 2011 \\
\hline P13 & Janssen, M. & $\begin{array}{l}\text { Exploring the service-oriented enterprise: } \\
\text { Drawing lessons from a case study }\end{array}$ & 2008 \\
\hline $\mathrm{P} 14$ & $\begin{array}{l}\text { Nantaburom, K., Chan, H., } \\
\text { Chung, S., Chan, F. T. S. }\end{array}$ & $\begin{array}{l}\text { Impact of Service-Oriented Architecture on } \\
\text { service supply chains }\end{array}$ & 2010 \\
\hline P15 & $\begin{array}{l}\text { Blanton, J., Leski, S., } \\
\text { Nicks, B., Tirzaman, T. }\end{array}$ & Making SOA work in a healthcare company & 2009 \\
\hline P16 & Keller, T., Dietrich, M. & $\begin{array}{l}\text { Management of SOA in public administration: A } \\
\text { case study }\end{array}$ & 2009 \\
\hline P17 & $\begin{array}{l}\text { Eckert, J., Bachhuber, M., } \\
\text { Miede, A., Papageorgiou, } \\
\text { A., Steinmetz, R. }\end{array}$ & $\begin{array}{l}\text { Readiness and Maturity of Service-oriented } \\
\text { Architectures in the German Banking Industry }\end{array}$ & 2010 \\
\hline P18 & Haines, M., Haseman, W. & Service-Oriented Architecture Adoption Patterns & 2009 \\
\hline P19 & Luthria, H., Rabhi, F. A. & Service-Oriented Architectures: Myth or Reality? & 2012 \\
\hline $\mathrm{P} 20$ & Löhe, J., Legner, C. & $\begin{array}{l}\text { SOA adoption in business networks: Do service- } \\
\text { oriented architectures really advance inter- } \\
\text { organizational integration? }\end{array}$ & 2010 \\
\hline $\mathrm{P} 21$ & $\begin{array}{l}\text { Trkman, P., Kovacic, A., } \\
\text { Popovic, A. }\end{array}$ & SOA adoption phases: A case study & 2011 \\
\hline $\mathrm{P} 22$ & Krushna, R., Ranjan, M. & $\begin{array}{l}\text { SOA for e-Governance in India: Potentials and } \\
\text { pitfalls }\end{array}$ & 2009 \\
\hline $\mathrm{P} 23$ & Bhallamudi, P., Tilley, S. & SOA migration case studies and lessons learned & 2011 \\
\hline $\mathrm{P} 24$ & Geric, $\mathrm{S}$. & The potential of service-oriented architectures & 2010 \\
\hline P25 & Baskerville, R. et al. & $\begin{array}{l}\text { The strategic value of SOA: A comparative case } \\
\text { study in the banking sector }\end{array}$ & 2010 \\
\hline P26 & $\begin{array}{l}\text { Joachim, N., Beimborn, } \\
\text { D., Weitzel, T. }\end{array}$ & $\begin{array}{l}\text { What Are Important } \\
\text { Management Mechanisms to } \\
\text { Flexibility in Service-Oriented Achieve IT } \\
\text { (SOA)?: An Empirical Exploration }\end{array}$ & 2011 \\
\hline P27 & $\begin{array}{l}\text { Ebert, N., Hochstein, A., } \\
\text { Brenner, W. }\end{array}$ & $\begin{array}{l}\text { Where to Start with SOA: Criteria for Selecting } \\
\text { SOA Projects }\end{array}$ & 2008 \\
\hline
\end{tabular}

